Pacific Journal of Mathematics

A NOTE ON BANACH SPACES OF LIPSCHITZ FUNCTIONS 


\title{
A NOTE ON BANACH SPACES OF LIPSCHITZ FUNCTIONS
}

\author{
JERRY JOHNSON
}

This note is divided into two sections. The first establishes some properties of extreme Lipschitz functions that, it is hoped, will lead to satisfactory ways of characterizing them in general. The second section shows how ideas due to Lindenstrauss can be used to establish the existence of Lipschitz spaces that fail to be injective and fail the approximation property.

Introduction. Our notation will follow essentially that of [6] and [11]. Given a metric space $(S, d), \operatorname{Lip}(S, d)$ denotes the Banach space of bounded real-valued functions on $S$ with norm given by $\|f\|=\max \left(\|f\|_{\infty},\|f\|_{d}\right)$, where

$$
\|f\|_{d}=\sup \left\{|f(s)-f(t)| d^{-1}(s, t): s \neq t\right\}<\infty .
$$

The closed subspace of functions $f$ for which $|f(s)-f(t)|=o(d(s, t))$ is denoted by $\operatorname{lip}(S, d)$. If $A \subset S, \tilde{A}$ denotes its complement in $S$, and if $f: S \rightarrow R$ is a function, $M_{f}$ denotes $\left\{s:|f(s)|=\|f\|_{\infty}\right\}$.

In [11], Roy showed that a function $f$ is an extreme point of the unit ball of $\operatorname{Lip}(S, d)$, with $S$ the unit interval and $d$ the usual metric, if and only if $\left|f^{\prime}\right|=1$ a.e. on $\tilde{M}_{f}$ and $\|f\|=\|f\|_{\infty}=1$. (See [10] for more along these lines.) The purpose of $\S 1$ of this note is to discuss some results that we hope will provide clues to possible characterizations of these extreme points for more general metric spaces by presenting two ways in which the bond imposed by the condition " $\left|f^{\prime}\right|=1$ a.e. on $\tilde{M}_{f}$ " may be broken.

In $\$ 2$ we observe that if $(S, d)$ is the unit ball of Enflo's space [4], then $\operatorname{Lip}(S, d)$ fails the approximation property.

We also show that $\operatorname{Lip}(S, d)$, where $(S, d)$ is the Hilbert cube, is not injective, ie., is not a $\mathscr{P}_{\lambda}$ space for any $\lambda$ (see [2, p. 94]). This last result uses techniques due to Lindenstrauss [8], and contrasts with the many examples where $\operatorname{Lip}(S, d)$ is isomorphic to the sequence space $l_{\infty}$. (See the discussion preceding Proposition 2.2.) The results of $\$ 2$ point out how large the class of $\operatorname{spaces} \operatorname{Lip}(S, d)$ is, although they are always dual spaces [6] and, for $S$ infinite, always contain a copy of $l_{\infty}$ [7].

1. We begin by giving a lemma, mainly for the sake of completeness, which is very similar to the technique of Phelps that was used in [11, p. 1159]. 
Lemma 1.1. Let $E$ be a Banach space, $F$ a dense subspace of $E$ and $U^{*}$ the unit ball of the dual $E^{*}$ of $E$. If $\sup \left\{x^{*}(x): x^{*} \in \mathscr{A}\right\}=\|x\|$ for all $x \in F$, then $U^{*}$ is the weak*-closed convex hull of $\mathscr{A}$.

Proof. Let $K$ be the weak*-closed convex hull of $\mathscr{A}$. If $x_{0}^{*} \in$ $U^{*} \sim K$, then there is some $x_{0}$ in $E$ with $\alpha=\sup \left\{x^{*}\left(x_{0}\right): x^{*} \in K\right\}<$ $x_{0}^{*}\left(x_{0}\right)=\beta$. Choose $y_{0} \in F \quad$ with $\left\|x_{0}-y_{0}\right\|<\frac{1}{2}(\beta-\alpha)$. Then $\sup \left\{x^{*}\left(y_{0}\right): x^{*} \in K\right\} \leqq \frac{1}{2}(\alpha+\beta)<x_{0}^{*}\left(y_{0}\right)$, a contradiction.

Proposition 1.1. Let $(S, d)$ be a compact, connected metric space. Let $\mathscr{A}$ denote the collection of all functions $f$ in the unit ball of $\operatorname{Lip}(S, d)$ with $\|f\|_{\infty}=1$ such that there is a finite nonempty set $P \subset S$ with the property that given any $s \in \tilde{M}_{f},|f(s)-f(t)|=d(s, t)$ for some $t \in P$. Then $\mathscr{A}$ is a sup-norm dense subset of the extreme points of the unit ball of $\operatorname{Lip}(S, d)$.

Proof. Let $f \in \mathscr{A}$ and suppose $\|f \pm g\| \leqq 1$. If $s \in \tilde{M}_{f} \cap \tilde{P}$ then there is some $t \in P$ such that $|\tilde{f}(s, t)|=1$ where $\tilde{f}(s, t)=$ $f(s)-f(t) / d(s, t)$. Since $|\tilde{f}(s, t) \pm \tilde{g}(s, t)| \leqq 1, \tilde{g}(s, t)=0$ and therefore $g(s)=g(t)$. If $s \in M_{f}, g(s)=0$. Hence $g(S)=\{0\} \cup g(P)$. Since $S$ is connected and $P$ is finite, $g(S)$ is a singleton. But $\|f\|_{\infty}=1$, so $0 \in g(S)$. Hence $g=0$ and $f$ is extreme. Thus $\mathscr{A}$ is a subset of the extreme points. Now, let $F$ denote the linear span of the point evaluations in the dual of $\operatorname{Lip}(S, d)$. As was shown in [6, p. 157] the dual of $F$ can be canonically identified with $\operatorname{Lip}(S, d)$ and $w^{*}$ convergence of bounded nets in $\operatorname{Lip}(S, d)$ is equivalent with uniform convergence. Hence, if we show that $\sup \{\phi(f): f \in \mathscr{A}\}=\|\phi\|$ for each $\phi \in F$, we will reach the desired conclusion by Lemma 1.1 and the $K^{2}-M^{3}-R$ theorem [2, p. 80]. To this end, let $\phi=\sum_{i=1}^{n} \lambda_{i} \epsilon_{s i}$. (Here $\epsilon_{s}: f \rightarrow f(s)$.) If $P=\left\{s_{1}, \cdots, s_{n}\right\}$, then clearly $\|\phi\| \leqq\left\|\phi_{\mid P}\right\|$. By [12, Prop. 1.4 ], we can extend each function in $\operatorname{Lip}(P, d)$, without increase of norm, to an element of $\operatorname{Lip}(S, d)$. Thus, $\|\phi\|=\left\|\left.\phi\right|_{p}\right\|$, where $\left.\phi\right|_{p}$ is short for $\left.\phi\right|_{\operatorname{Lip}(p, d)}$. Now $\phi_{\mid p}$ attains its norm on the unit ball of the finite dimensional space $\operatorname{Lip}(P, d)$ at an extreme point $g$. We extend $g$ in a norm preserving way to $S$ by the technique of Sherbert [12, Prop. 1.4] as follows: Let $g_{0}(s)=\max _{1 \leqq j \leqq n} g\left(s_{j}\right)-d\left(s, s_{j}\right)$ and let

$$
f(s)=\left\{\begin{array}{rll}
g_{0}(s) & \text { if } & -1 \leqq g_{0}(s) \leqq 1 \\
1 & \text { if } & g_{0}(s)>1 \\
-1 & \text { if } & g_{0}(s)<-1
\end{array}\right.
$$

Since $f$ extends $g$, we have $\|\phi\|=\left\|\phi_{\mid p}\right\|=\phi_{\mid p}(g)=\phi(f)$. If we show $f \in \mathscr{A}$, we are finished.

First, $\|f\| \leqq\|g\|=1$ and since $g$ is extreme $\|g\|_{\infty}=1$. Thus, $\|f\|_{\infty}=$ 
1. Let $s \in \tilde{M}_{f}$. Then $|f(s)|<1$, so $f(s)=g_{0}(s)=g\left(s_{j}\right)-d\left(s, s_{j}\right)$ for some $j=1, \cdots, n$. But $f=g$ on $P$, so $f(s)=f\left(s_{i}\right)-d\left(s, s_{j}\right)$ or $\left|f(s)-f\left(s_{j}\right)\right|=d\left(s, s_{j}\right)$. Hence, $f \in \mathscr{A}$. This completes the proof.

REMARK. Define $\mathscr{B}$ to be the set of all functions $f$ in the unit ball of $\operatorname{Lip}(S, d)$ such that there is a finite subset $P$ of $S$ with the property that for each $s \in S$, we have $|f(s)-f(t)|=d(s, t)$ for some $t \in M_{f} \cup P$. The same argument as above shows $f$ is an extreme point of the unit ball. It is not difficult to see that the function defined in [11, Lemma 1.3] is in $\mathscr{B}(P=\varnothing$ in this case) and that $\mathscr{A} \subset \mathscr{B}$.

Here we digress momentarily in order to show how the technique of the above proof can also be used to prove the following.

Proposition 1.2. Let $0<\alpha<1$ and $(S, d)$ be a compact metric space. Then $\cup_{\beta>\alpha} \operatorname{Lip}\left(S, d^{\beta}\right)$ is dense in $\operatorname{lip}\left(S, d^{\alpha}\right)$.

Proof. We apply the technique mentioned earlier ([11, p. 1159]). Let $\phi=\sum_{i=1}^{n} \lambda_{i} \epsilon_{s_{i}}$ be taken in the dual of $\operatorname{lip}\left(S, d^{\alpha}\right)$ with $\|\phi\|=$ 1. As in the proof of Proposition 1.1, we consider $\phi=\phi_{\mid p}$ as an element of $\operatorname{Lip}\left(P, d^{\alpha}\right)^{*}$ where $P=\left\{s_{1}, \cdots, s_{n}\right\}$. Its norm is attained at an extreme point $h$ of the unit ball. Given $\epsilon>0$, choose $\beta>\alpha$ sufficiently near $\alpha$ so that $\inf _{i \neq j} d^{\beta-\alpha}\left(s_{i}, s_{j}\right)$ and $(\operatorname{diam} S)^{\beta-\alpha}$ are greater than $1 /(1+\epsilon)$. Define $f_{0}$ by

$$
f_{0}(s)=\sup _{j} h\left(s_{j}\right)-d^{\beta}\left(s_{j}, s\right)
$$

and then let $f$ be the truncation of $f_{0}$ as in Proposition 1.1. Now, $\|h\|_{d^{\beta}} \leqq(1+\epsilon)\|h\|_{d^{\alpha}} \leqq 1+\epsilon$, by our choice of $\beta$. Since

$$
\frac{|f(s)-f(t)|}{d^{\alpha}(s, t)}=\frac{|f(s)-f(t)|}{d^{\beta}(s, t)} d^{\beta-\alpha}(s, t),
$$

$\|f\|_{d^{\alpha}} \leqq\|f\|_{d^{\beta}} p^{\beta-\alpha} \leqq\|h\|_{d^{\beta}}(1+\epsilon) \leqq(1+\epsilon)^{2}$ where $p$ is the diameter of $S$. Let $g=(1+\epsilon)^{-2} f$. Then $g$ belongs to $\operatorname{Lip}\left(S, d^{\beta}\right)$ and to the unit ball of $\operatorname{lip}\left(S, d^{\alpha}\right)$. Also $\phi(g)=(1+\epsilon)^{-2} \phi(f)$ and $\phi(f)=\phi(h)=\left\|\phi_{\mid p}\right\|=$ $\|\phi\|$ taken in $\operatorname{Lip}\left(S, d^{\alpha}\right)^{*}$. It follows from lemma 4.6 in [6] that the norm of $\phi$ taken in $\operatorname{Lip}\left(S, d^{\alpha}\right)^{*}$ is equal with its value in $\operatorname{lip}\left(S, d^{\alpha}\right)^{*}$. Therefore, $\phi(g)=(1+\epsilon)^{-2}$. But $\epsilon>0$ was arbitrary, so $1=\|\phi\|=\sup \phi(g)$ where the supremum is taken over all $g$ in the unit ball of $\operatorname{lip}\left(S, d^{\alpha}\right)$ that lie in $\cup_{\beta>\alpha} \operatorname{Lip}\left(S, d^{\beta}\right)$. It was proved by Jenkins [5] that the point evaluations span a norm dense subspace of $\operatorname{lip}\left(S, d^{\alpha}\right)^{*}$. Hence the unit ball of $\operatorname{lip}\left(S, d^{\alpha}\right)^{*}$ is the norm-closed convex hull of the subset consisting of members of $U_{\beta>\alpha}$ $\operatorname{Lip}\left(S, d^{\beta}\right)$. This completes the proof. 
The last result we include in this section is not very satisfying since we have been unable to establish it for metric spaces more general than an interval and also since it cannot, at least in its present form, be made to work if $\operatorname{lip}(S, d)$ is nontrivial (as an examination of the proof will show). The only reason we include it is that, as mentioned in the introduction, there have to our knowledge been no characterizations of extreme Lipschitz functions, other than that of Roy [11], and no real clues as to how to extend his characterization. We only hope that the next proposition, as well as Proposition 1.1, may give some ideas as to possible directions to take.

In what follows $\mathscr{D}_{s}$ denotes the set of point derivations at $s$ in $\operatorname{Lip}(S, d)^{*}($ See $[12, \S 8])$. We now turn to

Proposition 1.3. Let $f$ be in the unit ball of $\operatorname{Lip}(I, d)$, with $\|f\|_{\infty}=1$, where $I$ is the unit interval and $d$ the usual metric. Then $f$ is extreme if and only if the weak ${ }^{*}$ closure of the linear span of $\cup_{s \notin M_{f}}$ $\left\{\phi \in \mathscr{D}_{s}:\|\phi\|=|\phi(f)|=1\right\}$ contains $\bigcup_{s \notin M_{f}} \mathscr{D}_{s}$.

Proof. Suppose the condition holds. If $\|f \pm g\| \leqq 1$, then $\phi(g)=$ 0 for all $\phi \in \bigcup_{s \notin M_{f}} \mathscr{D}_{s}$. Hence, by [12, Prop. 9.10], $g$ is in $\operatorname{lip}(A, d)$ for each component $A$ of $\tilde{M}_{f}$ and is therefore constant on each $A$. Since $g=0$ on $M_{f}, g=0$ on $I$. Thus, $f$ is extreme. Now assume $f$ is extreme. Then by Roy's characterization [11, Theorem 3.1], $\left|f^{\prime}\right|=1$ a.e. on $\tilde{M}_{f}$. Let $\mathcal{M}_{s}$ denote the set of multiplicative linear functionals $x^{*}$ in the dual of $L_{\infty}(I)$ such that $x^{*}(g)=g(s)$ for each continuous function $g$ on $I$. If $D: \operatorname{Lip}(I, d) \rightarrow L_{\infty}(I)$ is defined by $D f=f^{\prime}$ (to be precise, the equivalence class containing $\left.f^{\prime}\right)$, then $D^{*} \mathcal{M}_{s}$ is contained in $\left\{\phi \in \mathscr{D}_{s}:\|\phi\|=|\phi(f)|=1\right\}$ for each $s \notin M_{f}$; here $D^{*}$ denotes the adjoint of $D$. If we show $\cup_{s \notin M_{f}} D^{*} \mathcal{M}_{s}$ is weak* dense in $\bigcup_{s \notin M_{f}} \mathscr{D}_{s}$ we will be finished. Suppose $f \in \operatorname{Lip}(I, d)$ and $\phi(f)=0$ for each $\phi \in \bigcup_{s \notin M_{f}} D^{*} \mathcal{M}_{s}$. Then $x^{*}\left(f^{\prime}\right)=0$ for each $x^{*} \in \mathcal{M}_{s}, s \notin M_{f}$. We will show $f^{\prime}=0$ a.e. on $\tilde{M}_{f}$. Given $\epsilon>0$, there is a compact $K \subset \tilde{M}_{f}$ with $\mu\left(\tilde{M}_{f} \sim K\right)<\epsilon(\mu=$ Lebesgue measure on $I$. $) \quad$ Let $h$ be continuous on $I$ with $h=1$ on $K$ and $h=0$ on $M_{f}$. Then $x^{*}\left(f^{\prime} h\right)=0$ for each $x^{*} \in \cup_{s \in I} M_{s}$, the whole maximal ideal space of $L_{\infty}(I)$. Thus, $f^{\prime} h=0$ a.e., so $f^{\prime}=0$ a.e. on $K$. But $\mu\left(\tilde{M}_{f} \sim K\right)<\epsilon$, so $\mu\left\{s \in \tilde{M}_{f}: f^{\prime}(s) \neq 0\right\}<$ $\epsilon$. Hence $f^{\prime}=0$ a.e. on $\tilde{M}_{f}$ and thus $f$ is constant on each component of $\tilde{M}_{f}$. Now, if $\phi \in \mathscr{D}_{s}$ and $s \in \tilde{M}_{f}$, then $f$ is constant in a neighborhood of $s$ and thus $\phi(f)=0$ for each $\phi \in \mathscr{D}_{s}$. Since $\phi(f)=0$ for all $\phi \in \cup_{s \notin \dot{M_{f}}} D^{*} \mathcal{M}_{s}$ implies $\phi(f)=0$ for all $\phi \in \bigcup_{s \notin M_{f}} \mathscr{D}_{s}$, we conclude that the former is weak*-dense in the latter. This completes the proof.

Let us remark that the above proposition holds for complex scalars. We are forced to use real scalars in Proposition 1.1 since our norm preserving extensions are not available otherwise without extra assumptions on $(S, d)$ such as the Lipschitz 4-point property (see [5]). 
2. In this section we present two applications of the paper of Lindenstrauss [8] which indicate just how general the class of spaces $\operatorname{Lip}(S, d)$ is.

The first is a relatively straightforward application of two powerful theorems to give the following.

Proposition 2.1. Let $E$ be a Banach space without the approximation property (see [4], of course) and let $S$ denote its unit ball with $d$ given by the norm. Then $\operatorname{Lip}(S, d)$ fails the approximation property.

Proof. Let $\phi(x)=x$ if $x \in S$ and $\phi(x)=x /\|x\|$ if $x \notin S$. Then $\|\phi(x)-\phi(y)\| \leqq 2\|x-y\|$ for each $x, y \in E$. To see this, consider the cases (1) $x, y \notin S$ and (2) $x \in S, y \notin S$.

$$
\begin{aligned}
& \|\phi(x)-\phi(y)\| \leqq\left\|\frac{x}{\|x\|}-\frac{x}{\|y\|}\right\|+\left\|\frac{x}{\|y\|}-\frac{y}{\|y\|}\right\| \\
& =\frac{\mid\|y\|-\|x\|}{\|y\|}+\frac{1}{\|y\|}\|x-y\| \leqq \frac{2}{\|y\|}\|x-y\| \leqq 2\|x-y\| .
\end{aligned}
$$

$$
\begin{aligned}
& \|\phi(x)-\phi(y)\| \leqq\left\|x-\frac{x}{\|y\|}\right\|+\left\|\frac{x}{\|y\|}-\frac{y}{\|y\|}\right\| \\
& \leqq \frac{\|y\|-1}{\|y\|}+\frac{\|x-y\|}{\|y\|} \leqq \frac{\|y\|-\|x\|}{\|y\|}+\frac{\|x-y\|}{\|y\|} \\
& \leqq \frac{2}{\|y\|}\|x-y\| \leqq 2\|x-y\| .
\end{aligned}
$$

Now, $T: f \rightarrow f \circ \phi$ is easily seen to be an isomorphism (= linear homeomorphism) of $L_{0}$ into $E^{*}$ where $L_{0}=\{f \in \operatorname{Lip}(S, d): f(0)=0\}$ and $E^{*}=\left\{f: E \rightarrow R:\|f\|_{d}<\infty, f(0)=0\right\}$. Let $Q$ be the restriction mapping of $E^{*}$ into $\operatorname{Lip}(S, d), P$ the projection of $E^{*}$ on $E^{*}$ guaranteed by [8, Theorem 2], and $J$ the mapping of $\operatorname{Lip}(S, d)$ into $L_{0}$ defined by $J f=f-f(0)$. Then $Q P T J$ is a projection of $\operatorname{Lip}(S, d)$ on $Q E^{*}$. Hence $E^{*}$ is isomorphic to a complemented subspace of $\operatorname{Lip}(S, d)$. Since $E$ fails the approximation property, so does $E^{*}$ and hence $\operatorname{Lip}(S, d)$. This completes the proof.

We have as yet been unable to prove or disprove the existence of a compact metric space for which $\operatorname{Lip}(S, d)$ fails the approximation property. For a discussion of reformulations of this problem, see $[6, p$. 168].

We now turn our attention to another application of [8] in which we show that $\operatorname{Lip}(S, d)$, with $(S, d)$ the Hilbert cube, is not injective. This contrasts with the case where $(S, d)$ is the unit interval with the usual 
metric and the case where $(S, d)$ is an infinite compact subset of Euclidean space with $d=|\cdot|^{\alpha}, 0<\alpha<1$. In all these situations $\operatorname{Lip}(S, d)$ is isomorphic to the sequence space $l_{\infty}$ and is hence injective. (For $0<\alpha<1$, see [1]; for the interval and $\alpha=1$, the assertion follows from [9] and the fact that $\|f\|_{d}=\left\|f^{\prime}\right\|_{\infty}$ in $L_{\infty}(I)$.)

Let us remark that it is not hard to show in addition that $\operatorname{Lip}(S, d)$ is isomorphic to $l_{\infty}$ for any compact infinite subset of the line. Simply let $I$ be a closed interval with $S$ in its interior. Then for each $f$ in $\operatorname{Lip}(S, d$ let $T f$ be the function obtained by extending $f$ linearly in each component of $I \sim S . \quad T$ is an isomorphism of $\operatorname{Lip}(S, d)$ into $\operatorname{Lip}(I, d)$ whose composition $T R$ with the restriction map is a projection on $T \operatorname{Lip}(S, d)$. But it is a well known fact due to Lindenstrauss that a complemented infinite dimensional subspace of $l_{\infty}$ is isomorphic to $l_{\infty}$.

It is tempting to conjecture that if $(S, d)$ is a compact subset of Euclidean space, $\operatorname{Lip}(S, d)$ is injective. We have been unable to prove it however.

The proof of Proposition 2.2 is broken down into two lemmas. Lemma 2.1 is certainly well-known and is even a corollary to far stronger results. We sketch a proof only for completeness.

Lemma 2.1. An infinite dimensional reflexive subspace $E$ of the continuous functions $C(X)$ on a compact Hausdorff space $X$ is not complemented in $C(X)$.

Proof. If $P$ is a projection of $C(X)$ on $E, P$ is weakly compact because $E$ is reflexive. By [3, Corollary VI. 7.5], $P$ is compact. But $P$ is onto $E$, so $E$ is finite dimensional.

In the sequel, $C$ will denote the continuous functions on $[0,1], E$ an isometric copy of $l_{2}$ in $C$, and $(S, d)$ the Hilbert cube in $E^{*}=E$.

Lemma 2.2. There is no map $\alpha: S \rightarrow C^{*}$ and no constant $M>0$ such that $\alpha\left(x^{*}\right)_{\mid E}=x^{*}$ and $\left\|\alpha\left(x^{*}\right)-\alpha\left(y^{*}\right)\right\| \leqq M\left\|x^{*}-y^{*}\right\|$ for each $x^{*}, y^{*} \in S$.

Proof. (The idea for this proof is due to Professor Joram Lindenstrauss, to whom I express my thanks for permission to include it here.)

Suppose such a mapping exists. We will construct a map $\beta: E^{*} \rightarrow C^{*}$ with the same properties. First, we may assume, by replacing $\alpha$ by $\alpha-\alpha(0)$, that $\alpha(0)=0$. Let $K_{x}^{*}=$ $\{0\} \cup\left\{\mu \in C^{*} \mid \mu_{\mid E}=x^{*}\right.$ and $\left.\|\mu\| \leqq M\left\|x^{*}\right\|\right\}$ and define

$$
\alpha_{n}\left(x^{*}\right)=\left\{\begin{array}{ccc}
n \alpha\left(\frac{1}{n} x^{*}\right) & \text { if } & x^{*} \in n S \\
0 & \text { if } & x^{*} \notin n S
\end{array} .\right.
$$


If $\Pi$ is the product of those $K_{x}^{*}$ 's with $x^{*} \in \cup_{n} n S$, then $\alpha_{n} \in \Pi$ for each $n$. In fact, for $x^{*}, y^{*} \in n S$, we have

$$
\begin{aligned}
& \left\|\alpha_{n}\left(x^{*}\right)-\alpha_{n}\left(y^{*}\right)\right\| \\
& \quad=\left\|n \alpha\left(\frac{1}{n} x^{*}\right)-n \alpha\left(\frac{1}{n} y^{*}\right)\right\| \leqq n M\left\|\frac{1}{n} x^{*}-\frac{1}{n} y *\right\| \\
& \quad=M\left\|x^{*}-y^{*}\right\| .
\end{aligned}
$$

Now, each $K_{x}^{*}$ is weak*-compact, so $\Pi$ is compact in the product topology. Thus, there is a subnet $\left\{\alpha_{n_{\gamma}}\right\}$ of $\left\{\alpha_{n}\right\}$ converging to some member $\beta$ of $\Pi$. Let $x^{*}, y^{*} \in \cup n S$. Pick $n_{0}$ so that $x^{*}, y^{*} \in$ $n_{0} S$. Then there is an index $\gamma_{0}$ such that $n_{\gamma} \geqq n_{0}$ for all $\gamma \geqq \gamma_{0}$. If $x \in C, \quad\|x\| \leqq 1$, we have $\left.\mid \beta\left(x^{*}\right)-\beta\left(y^{*}\right)\right)(x)\left|=\lim _{\gamma \geqq \gamma_{0}}\right|\left(\alpha_{n_{\gamma}}\left(x^{*}\right)-\right.$ $\left.\alpha_{n_{\gamma}}\left(y^{*}\right)\right)(x) \mid$. But $n_{\gamma} \geqq n_{0}$ for $\gamma \geqq \gamma_{0}$ and $S$ is circled,so $x^{*}, y^{*} \in n_{\gamma} S$ for $\gamma \geqq \gamma_{0}$. Thus, $\left\|\alpha_{n_{\gamma}}\left(x^{*}\right)-\alpha_{n_{\gamma}}\left(y^{*}\right)\right\| \leqq M\left\|x^{*}-y^{*}\right\|$ for each $\gamma \geqq \gamma_{0}$, and $\left\|\beta\left(x^{*}\right)-\beta\left(y^{*}\right)\right\| \leqq M\left\|x^{*}-y^{*}\right\|$. Now, $\cup n S$ is dense in $E^{*}$, so $\beta$ extends uniquely to all of $E^{*}$ and $\left\|\beta\left(x^{*}\right)-\beta\left(y^{*}\right)\right\| \leqq M\left\|x^{*}-y^{*}\right\|$ for all $x^{*}, y^{*} \in E^{*}$. Since $\beta=\lim _{\gamma} \alpha_{n_{\gamma}}$, we have $\beta\left(x^{*}\right)_{\mid \mathrm{E}}=x^{*}$ for each $x^{*} \in \cup n S$. But $\beta$ is continuous on $E^{*}$ and $\cup n S$ is dense in $E^{*}$ so $\beta\left(x^{*}\right)_{\mid E}=x^{*}$ for all $x^{*} \in E^{*}$. Hence $\beta$ is the required mapping. Now, define $T: C^{* *} \rightarrow E^{* *}$ by $T F\left(x^{*}\right)=F\left(\beta x^{*}\right)$ for $F \in C^{* *}, x^{*} \in E^{*}$ (see the proof of Proposition 2.1 for the definition of $\left.A^{*}\right)$. $\quad T$ is linear and continuous and by [8, Theorem 2] there is a projection $P$ of $E^{* *}$ onto $E^{* *}$. Consider $P T_{\mid c}: C \rightarrow E^{* *}$. If $x \in E$, let $F_{x}(\mu)=\mu(x)$ for each $\mu \in C^{*}$. Then $T F_{x}\left(x^{*}\right)=F_{x}\left(\beta x^{*}\right)=$ $\left(\beta x^{*}\right)(x)=x^{*}(x)$. This says $T F_{x}=F_{x}$, so $P T F_{x}=F_{x}$. Thus, identifying $E$ and $E^{* *}$, we obtain a projection of $C$ onto $E$, a contradiction.

Proposition 2.2. If $(S, d)$ is the Hilbert cube, $\operatorname{Lip}(S, d)$ is not injective.

Proof. A necessary and sufficient condition for a Banach space $A$ to be injective is that for each Banach space $F$, subspace $F_{0} \subset F$, and bounded linear operator $T: F_{0} \rightarrow A$, there is a bounded linear operator $\tilde{T}: F \rightarrow A$ with $\tilde{T}_{\mid F_{0}}=T$ (see [2, p. 94]). We continue to use the notation of Lemma 2.2. Let $T: E \rightarrow \operatorname{Lip}(S, d)$ be defined by $T x: x^{*} \rightarrow x^{*}(x), x^{*} \in S$. If $\operatorname{Lip}(S, d)$ is injective, there is an operator $\tilde{T}: C \rightarrow \operatorname{Lip}(S, d)$ that extends $T$. Let $\alpha: S \rightarrow C^{*}$ be given by $\alpha\left(x^{*}\right): x \rightarrow(\tilde{T} x)\left(x^{*}\right)$. Then $\alpha$ satisfies the conditions in Lemma 2.2, an impossibility. This completes the proof.

Acknowledgement. The author thanks Professor Doug Hale for helpful conversations. 


\section{REFERENCES}

1. R. Bonic, J. Frampton, and A. Tromba, $\Lambda$-manifolds, J. Funct. Anal., 3 (1969), 310-320.

2. M. M. Day, Normed linear spaces, Ergebnisse Math., Academic Press, New York, 1962.

3. N. Dunford, and J. Schwartz, Linear operators, Part I, Interscience, New York, 1958.

4. Per Enflo, A counterexample to the approximation problem, Acta Math., 130 (1973), 309-317.

5. T. M. Jenkins, Banach spaces of Lipschitz functions on an abstract metric space, Thesis, Yale Univ., New Haven, Conn., 1967.

6. J. A. Johnson, Banach spaces of Lipschitz functions and vector-valued Lipschitz functions, Trans. Amer. Math. Soc., 148 (1970), 147-169.

7. Lipschtiz function spaces for arbitrary metrics, Bull. Amer. Math. Soc., 78 (1972), 702-705.

8. J. Lindenstrauss, On nonlinear projections in Banach spaces, Michigan Math. J., 11 (1964), 263-287.

9. A. Pelczynski, On the isomorphism of the spaces $H$ and $m$, Bull. Acad. Polon. Sci., 6 (1958), 695-696.

10. N. V. Rao, and A. K. Roy, Extreme Lipschitz functions, Math. Ann., 189 (1970), 26-46.

11. A. K. Roy, Extreme points and linear isometries of the Banach space of Lipschitz functions, Canad. J. Math., 20 (1968), 1150-1164.

12. D. R. Sherbert, The structure of ideals and point derivations in Banach algebras of Lipschitz functions, Trans. Amer. Math. Soc., 111 (1964), 240-272.

Received February 15, 1974. Supported in part by the O.S.U. College of Arts and Sciences Office of Research and Graduate Studies.

OKlahoma State University 


\section{PACIFIC JOURNAL OF MATHEMATICS}

\section{EDITORS}

RICHARD ARENS (Managing Editor)

University of California

Los Angeles, California 90024

\section{J. DugundJI}

Department of Mathematics University of Southern California Los Angeles, California 90007

D. Gilbarg and J. Milgram

Stanford University

Stanford, California 94305

\section{ASSOCIATE EDITORS}
E. F. BECKENBACH
B. H. NeumanN
F. WoLF
K. YoshiDA

\section{SUPPORTING INSTITUTIONS}

\author{
UNIVERSITY OF BRITISH COLUMBIA \\ CALIFORNIA INSTITUTE OF TECHNOLOGY \\ UNIVERSITY OF CALIFORNIA \\ MONTANA STATE UNIVERSITY \\ UNIVERSITY OF NEVADA \\ NEW MEXICO STATE UNIVERSITY \\ OREGON STATE UNIVERSITY \\ UNIVERSITY OF OREGON \\ OSAKA UNIVERSITY
}

\author{
UNIVERSITY OF SOUTHERN CALIFORNIA \\ STANFORD UNIVERSITY \\ UNIVERSITY OF TOKYO \\ UNIVERSITY OF UTAH \\ WASHINGTON STATE UNIVERSITY \\ UNIVERSITY OF WASHINGTON \\ AMERICAN MATHEMATICAL SOCIETY
}

The Supporting Institutions listed above contribute to the cost of publication of this Journal, but they are not owners or publishers and have no responsibility for its contents or policies.

Mathematical papers intended for publication in the Pacific Journal of Mathematics should be in typed form or offset-reproduced (not dittoed), double spaced with large margins. Underline Greek letters in red, German in green, and script in blue. The first paragraph or two must be capable of being used separately as a synopsis of the entire paper. Items of the bibliography should not be cited there unless absolutely necessary, in which case they must be identified by author and Journal, rather than by item number. Manuscripts, in duplicate, may be sent to any one of the four editors. Please classify according to the scheme of Math. Reviews, Index to Vol. 39. All other communications should be addressed to the managing editor, or Elaine Barth, University of California, Los Angeles, California, 90024.

100 reprints are provided free for each article, only if page charges have been substantially paid. Additional copies may be obtained at cost in multiples of 50 .

The Pacific Journal of Mathematics is issued monthly as of January 1966. Regular subscription rate: $\$ 72.00$ a year (6 Vols., 12 issues). Special rate: $\$ 36.00$ a year to individual members of supporting institutions.

Subscriptions, orders for back numbers, and changes of address should be sent to Pacific Journal of Mathematics, 103 Highland Boulevard, Berkeley, California, 94708.

PUBLISHED BY PACIFIC JOURNAL OF MATHEMATICS, A NON-PROFIT CORPORATION Printed at Jerusalem Academic Press, POB 2390, Jerusalem, Israel.

$$
\begin{gathered}
\text { Copyright (C) } 1975 \text { Pacific Journal of Mathematics } \\
\text { All Rights Reserved }
\end{gathered}
$$




\section{Pacific Journal of Mathematics}

\section{Vol. 58, No. $2 \quad$ April, 1975}

Zvi Artstein and John Allen Burns, Integration of compact set-valued functions . . . . . . . . . 297

Mark Benard, Characters and Schur indices of the unitary reflection group $[321]^{3} \ldots \ldots \ldots . .309$

Simeon M. Berman, A new characterization of characteristic functions of absolutely continuous

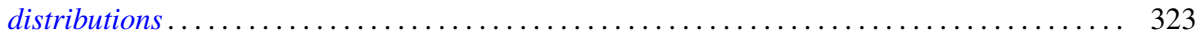

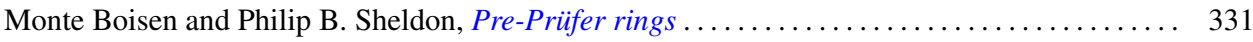

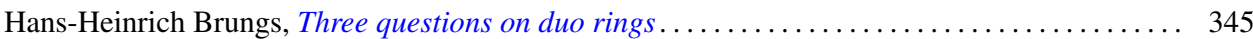

Iracema M. Bund, Birnbaum-Orlicz spaces of functions on groups................. 351

John D. Elwin and Donald R. Short, Branched immersions between 2-manifolds of higher

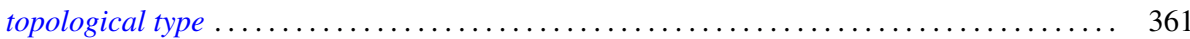

Eric Friedlander, Extension functions for rank 2, torsion free abelian groups . .......... 371

Jon Froemke and Robert Willis Quackenbush, The spectrum of an equational class of

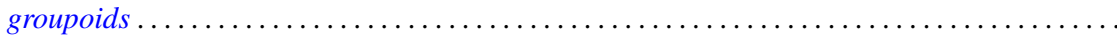

Barry J. Gardner, Radicals of supplementary semilattice sums of associative rings ...........

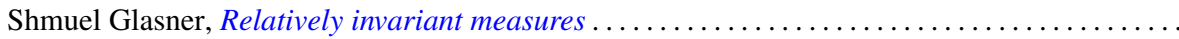

George Rudolph Gordh, Jr. and Sibe Mardesic, Characterizing local connectedness in inverse

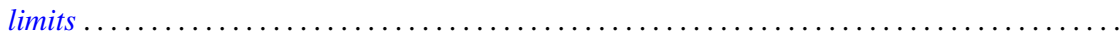

Siegfried Graf, On the existence of strong liftings in second countable topological spaces......

Stanley P. Gudder and D. Strawther, Orthogonally additive and orthogonally increasing

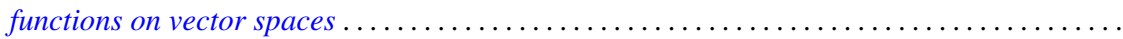

Darald Joe Hartfiel and Carlton James Maxson, A characterization of the maximal monoids and

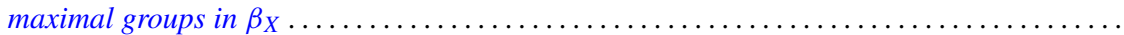

Robert E. Hartwig and S. Brent Morris, The universal flip matrix and the generalized faro-shuffle. .

William Emery Haver, Mappings between ANRs that are fine homotopy equivalences. .

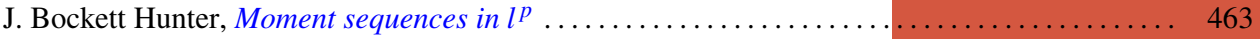

Barbara Jeffcott and William Thomas Spears, Semimodularity in the completion of a poset.... 467

Jerry Alan Johnson, A note on Banach spaces of Lipschitz functions . . . . . . . . . . . . 475

David W. Jonah and Bertram Manuel Schreiber, Transitive affine transformations on

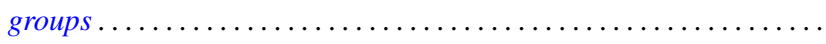

Karsten Juul, Some three-point subset properties connected with Menger's characterization of

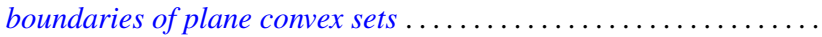

Ronald Brian Kirk, The Haar integral via non-standard analysis . . . . . . . . . . . . . 517

Justin Thomas Lloyd and William Smiley, On the group of permutations with countable

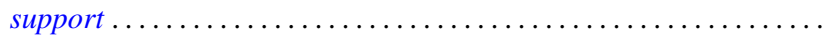

Erwin Lutwak, Dual mixed volumes .................................. 531

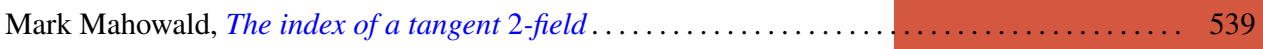

Keith Miller, Logarithmic convexity results for holomorphic semigroups . . . . . . . . . . . . 549

Paul Milnes, Extension of continuous functions on topological semigroups . . . . . . . . . . 553

Kenneth Clayton Pietz, Cauchy transforms and characteristic functions ................ 563

James Ted Rogers Jr., Whitney continua in the hyperspace $C(X) \ldots \ldots \ldots \ldots \ldots \ldots \ldots \ldots .569$

Jean-Marie G. Rolin, The inverse of a continuous additive functional . . . . . . . . . . . . 585

William Henry Ruckle, Absolutely divergent series and isomorphism of subspaces . ........ 605

Rolf Schneider, A measure of convexity for compact sets . ..................... 617

Alan Henry Schoenfeld, Continous measure-preserving maps onto Peano spaces .......... 627

V. Merriline Smith, Strongly superficial elements .......................... 643

Roger P. Ware, A note on quadratic forms over Pythagorean fields . . . . . . . . . . . . . . 651

Roger Allen Wiegand and Sylvia Wiegand, Finitely generated modules over Bezout rings . . . . 655

Martin Ziegler, A counterexample in the theory of definable automorphisms . . . . . . . . . 665 